\title{
Construcción de una mesa de succión de doble capa de bajo costo para definir macroporosidad (porosidad drenable) y microporosidad
}

Construction of a low-cost suction table to define macroporosity (drainable porosity) and microporosity

Adrián Enrique Chavarría-Vidal ${ }^{1}$, Jarbas Honorio de Miranda², Asdrubal Farias-Ramírez ${ }^{3}$

Chavarría-Vidal, A; Honorio de Miranda, J; Farias-Ramírez, A. Construcción de una mesa de succión de doble capa de bajo costo para definir macroporosidad (porosidad drenable) y microporosidad. Tecnología en Marcha. Vol. 33-3. JulioSetiembre 2020. Pág 90-97.

doi) https://doi.org/10.18845/tm.v33i3.4466

Fecha de recepción: 16 de julio de 2019

Fecha de aprobación: 11 de octubre de 2019

1 Profesor Escuela Ingeniería Agrícola, Instituto Tecnológico de Costa Rica, Estudiante doctorado Ciencias Naturales para el Desarrollo. Costa Rica. Correo electrónico: adchavarria@tec.ac.cr

2 Profesor Engenharia em Biossistemas, Escola Superior de Agricultura Luis de Queiroz Universidade de São Paulo. Brasil. 


\section{Palabras clave}

Macro porosidad; micro porosidad; porcentaje humedad gravimétrica; porcentaje de humedad volumétrica.

\section{Resumen}

La necesidad de conocer la porosidad drenable del suelo o macroporosidad con fines de manejar correctamente el agua en el suelo, sea ésta con fines de riego o con fines de drenaje ha tomado cada vez mayor relevancia. Para ello existen equipos que se ofrecen en casas comerciales generalmente de costos altos. No obstante, conociendo la física del agua en una matriz arenosa se puede construir una mesa de succión (mesa de tensión) con arena llamada comúnmente caja de arena para estimar la macro y microporosidad y como tal la porosidad total presente en un suelo.

Las micro porosidades obtenidas para un suelo arenoso expresado en contenido de humedad volumétrica sometido a una succión de $60 \mathrm{cmca}$ (centímetros de columna de agua) y a 100 cmca fueron $11,12 \%$ y $5,73 \%$ respectivamente y las macro porosidades obtenidas y expresadas en términos de porcentaje de humedad volumétrica para un suelo arenoso sometido a una succión de 60 cmca y a 100 cmca fueron $25,14 \%$ y 30,53\%.

\section{Keywords}

Macro porosity; micro porosity; gravimetric humidity percentage; volume humidity percentage.

\section{Abstract}

The need to know the drainable porosity of the soil or macro porosity in order to correctly manage the water in the soil, whether it is for irrigation purposes or for drainage purposes, has taken on increasing relevance. For this there are equipment that is offered in commercial houses, usually with high costs. However, knowing the physics of water in a sandy matrix, you can build a suction table (tension table) with sand commonly called sandbox to estimate the macro and micro porosity and as such the total porosity present in a soil.

The micro porosity obtained for a sandy soil subjected to a suction of $60 \mathrm{cmca}$ (centimeters of water column) and $100 \mathrm{cmca}$ were $11,12 \%$ and $5,73 \%$ of gravimetric humidity and the macro porosity obtained and expressed in terms of percentage of gravimetric humidity $\left(\left(g_{\text {water }} / g_{\text {soil }}\right)\right.$ * 100) for a sandy soil subjected to a suction of $60 \mathrm{cmca}$ and $100 \mathrm{cmca}$ were $25,14 \%$ and 30,53\%.

\section{Introducción}

La capacidad de un suelo para almacenar y transmitir agua son propiedades que inciden en su calidad y el sistema poroso es el responsable de ambas acciones [1].

A la razón del volumen total de vacíos $(\mathrm{Vv})$ que se presenta en un suelo y el volumen total de dicho suelo (VT) se define con el nombre de porosidad total del suelo (P) [2]. Por ello a la porosidad total del suelo se le llama índice de volumen relativo de poros en el suelo con relación al volumen total [3].

$$
\% P=\frac{V v}{V T} * 100 \ldots \ldots . .1
$$


Además, la porosidad total del suelo puede calcularse, a partir de la densidad real (Dr) o peso específico del suelo y densidad aparente del suelo (Da) o peso específico aparente del suelo por medio de la siguiente relación [4]:

$$
\% P=\left(1-\frac{D a}{D r}\right) * 100
$$

La porosidad se ha subdividido en varios tipos y para ello existen varias clasificaciones como lo son la porosidad según sus diferentes tamaños, según su funcionalidad con respecto al comportamiento hidráulico y los tamaños de diversos organismos vivos y las raíces. Para el caso del tipo de poros según su comportamiento hidráulico se clasifican en poros comunicantes (poros que funcionan de almacenamiento y drenaje) y no comunicantes (poros residuales) donde, los primeros se encuentran entre una succión de 0 - 1500 kPa (kilo pascales) de tensión de agua en el suelo y los segundos son todos los poros que se encuentran a una succión menor a $1500 \mathrm{kPa}$. Los poros comunicantes a su vez, se clasifican en poros de drenaje que son todos aquellos poros que se presentan entre una succión de 0 - $10 \mathrm{kPa}$, los poros de almacenamiento que se presentan entre una succión de $10 \mathrm{kPa}$ - $1500 \mathrm{kPa}$ y los poros residuales que se presentan a una succión $>1500$ kPa [1]. Además, el mismo autor menciona que Capacidad de Campo se presenta a una succión de $10 \mathrm{kPa}(100 \mathrm{cmca})$ y el Punto de Marchitez Permanente se presenta a una succión de $1500 \mathrm{kPa}(15000 \mathrm{cmca})$.

Según la clasificación por tamaño de los poros se clasifican en macroporos que son todos los poros mayores a $0,08 \mathrm{~mm}$ y los microporos que son todos aquellos poros menores a 0,08 mm. Los macroporos generalmente se encuentran entre las unidades estructurales de drenaje del agua gravitacional y que transmiten el aire de manera eficiente. Los microporos por el contrario, el movimiento relativo de aire y de agua es muy lento, y se subdividen en mesoporos $(0,03-0,08$ $\mathrm{mm}$ ) que son aquellos poros que quedan llenos de agua después de drenar libremente pero que tienen un movimiento de agua capilar y es disponible para las plantas, microporos (0,005 - 0,03 mm) que son aquellos que se encuentran dentro de las unidades estructurales del suelo y retienen agua disponible para las plantas, ultramicroporos $(0,0001-0,005 \mathrm{~mm})$ que retienen agua no disponible para las plantas y los criptoporos que son aquellos $<0,0001 \mathrm{~mm}$ [5].

De lo anterior podemos afirmar que:

\section{Porosidad total $=$ microporosidad + macroporisidad $\ldots \ldots \ldots 3$ \\ Macroporosidad = Porosidad total - Microporosidad ........4}

La microporosidad puede ser definida como el volumen de poros del suelo que posee un diámetro menor que 0,050 mm $(50 \mu \mathrm{m})$, lo cual corresponde al agua remanente después de ser el suelo drenado de los macroporos cuando se logra un equilibrio de una columna de agua de 0,60 m. Por ello la microporosidad corresponde al volumen de agua que ocupa un suelo después de alcanzar el equilibrio cuando es sometido a un potencial de 0,60 m que [6]. Además, el mismo autor define los diferentes tamaños de poros de la siguiente manera: los macroporos son todos los poros con un diámetro equivalente $>300 \mu \mathrm{m}$ y una columna de succión de agua $\leq$ 0,1 mca, mesoporos son todos los poros con un diámetro equivalente 300 - 50 um y una columna de succión de agua 0,1 - 0,6 mca, los microporos son todos los poros con un diámetro equivalente 50 - 0,2 $\mu \mathrm{m}$ y una columna de succión de agua 0,6 - 150 mca y los criptoporos son todos los poros con un diámetro equivalente $<0,20 \mu \mathrm{m}$ y una columna de succión de agua > 150 mca. 
Se puede observar que existe una relación entre el diámetro del poro equivalente y la succión de agua en el suelo cuyo nombre es la ecuación de capilaridad, la cual; [6] la define de la siguiente manera:

$$
\phi=\frac{0,3}{h}
$$

Donde:

$\varphi$ : diámetro equivalente del poro $(\mathrm{cm})$

h: altura de columna de agua $(\mathrm{cm})$

Un concepto asociado a la macroporosidad es el de porosidad drenable (Pd). Esta última se define como el volumen de agua que es drenado libremente por unidad de volumen total del

suelo $\left(\mathrm{cm}^{3}{ }_{\text {agua }} / \mathrm{cm}_{\text {suelo }}^{3}\right)^{\star} 100$. De esta forma será [7]:

$$
P d=\frac{\text { Volumen de agua drenada libremente }}{\text { Volumen total de suelo }}
$$

Claro está, después de que un suelo drena libremente el entra en el índice de humedad de capacidad de campo (máximo contenido de humedad que retiene el suelo en contra de la fuerza de la gravedad) y por ello la porosidad drenable o macroporosidad (anterior ecuación) queda de la siguiente manera [8] [7]:

$$
P d=\theta s a t-\theta c c
$$

Por lo que:

$$
\text { Macroporosidad }=\text { Porosidad drenable } \ldots \ldots \ldots . . . .8
$$

Donde:

$\theta$ sat $=$ humedad a saturación del suelo (todos los macroporos y microporos llenos de agua)

$\theta \mathrm{cc}=$ humedad a capacidad de campo (todos los microporos llenos de agua)

Valores encontrados en la literatura de capacidad de campo y punto de marchitez permanente ( $\theta$ pmp) en suelos arenosos son $\theta c c=5 \%-7 \%$ y $\theta p m p=1 \%-3 \%$ [9], $\theta c c=9 \%$ y $\theta p m p=2 \%$ [10], $\theta \mathrm{cc}=9 \%$ y $\theta \mathrm{pmp}=2 \%[11]$

\section{Metodología}

Se adquirieron los materiales que fueron un tubo de $12 \mathrm{~mm}$ de diámetro de PVC, 4 codos de PVC de $12 \mathrm{~mm}, 3 \mathrm{~T}$ de $12 \mathrm{~mm}$ de PVC, pegamento para PVC, tela de porosidad fina, fajas plásticas, 1 flanyer, conexiones de la tubería de PVC al flanyer y una cuba de plástico grueso y pegamento araldite profissional.

El tubo de PVC se cortó en pequeños trozos para formar dos rectángulos que unidos forman un cuadrado como se muestra en la figura 1, de tal manera que pueda entrar dentro de la cuba. Las uniones fueron selladas totalmente con pegamento para PVC para evitar la entrada de aire como se muestra en la figura 2. En estos rectángulos se perforaron los tubos a lo largo cada 10,0 mm en cruz, con un diámetro constante de 1,0 mm. Los tubos perforados se envolvieron en una tela porosa de poros muy pequeños y fueron sujetas con gazas plásticas. Luego a la cuba se le hizo una perforación en el fondo y centro de la misma con un diámetro ligeramente mayor para instalar el flanger el cual se instaló y en el proceso de selló para evitar la entrada de aire 
al sistema con el pegamento araldite profissional. Luego se realizó la conexión del flanger con la tubería de PVC la cual queda dentro de la cuba y también, se le instaló en el lado de abajo de la cuba, el sistema de drenaje que va a dar la columna de agua según la altura de succión que se les aplicará a las muestras. El costo de materiales de construcción y mano de obra fue de \$110.00 lo cual representa una fracción del costo de las que se ofrecen en el mercado.

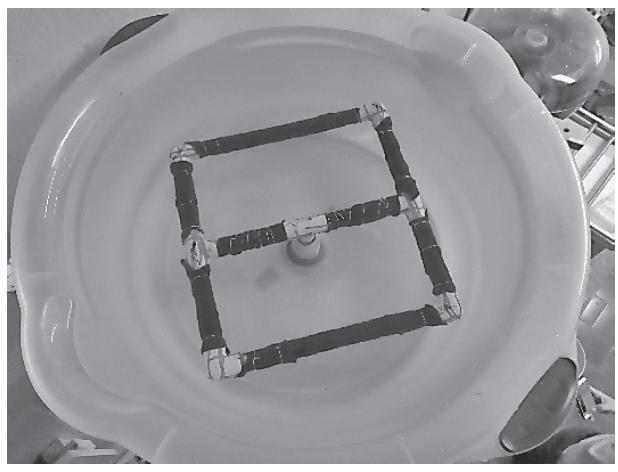

Figura. 1. tubería distribuidora de la presión de succión.

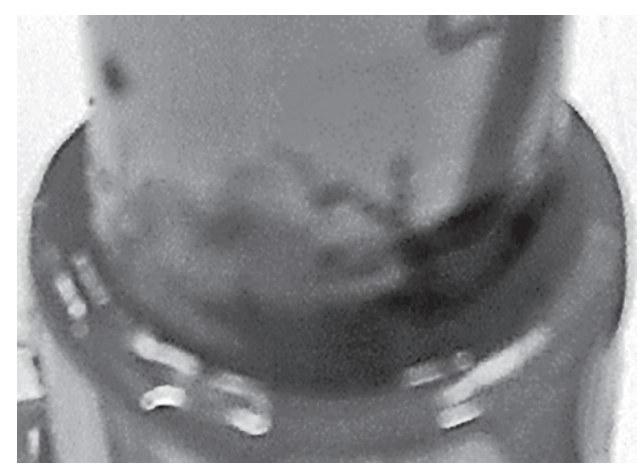

Figura 2. Sellado total de las uniones de PVC

Después de la construcción se procedió a saturar y depurar el sistema para sacar todo el aire que pudiera existir en el mismo ya que, las burbujas de aire tienen la capacidad de romper la tensión que ejerce la columna de agua

Como se muestra en la figura 3, se marcó la altura de succión cada $10 \mathrm{~cm}$ la cual es la referencia para controlar la tensión a aplicar a las muestras de suelo considerando que el nivel cero de succión se establece al mismo nivel de la altura máxima que queda la arena en la mesa de succión de bajo costo como se muestra en la figura 4.

Para lograr que la mesa de succión pueda ejercer una mayor succión de como mínimo 120 cm de columna de agua y cumplir con 60 o 100 cmca según el sistema de clasificación de macroporosidad usado, se utilizó doble capa de arena como se muestra en la figura 4. La primera capa de arena colocada en el fondo de la cuba fue un tamaño promedio de arena de 0,5 - 0,25 mm considerada como arena media y la segunda capa colocada la cual quedó en la superficie de la mesa de succión fue de 0,25 a 0,10 mm considerada como arena fina. La capa de arena media cubrió hasta con $5 \mathrm{~cm}$ de altura arriba de la tubería de PVC transportadora de la tensión ejercida como mínimo y la capa de arena fina fue de $5 \mathrm{~cm}$ como mínimo de grosor. 


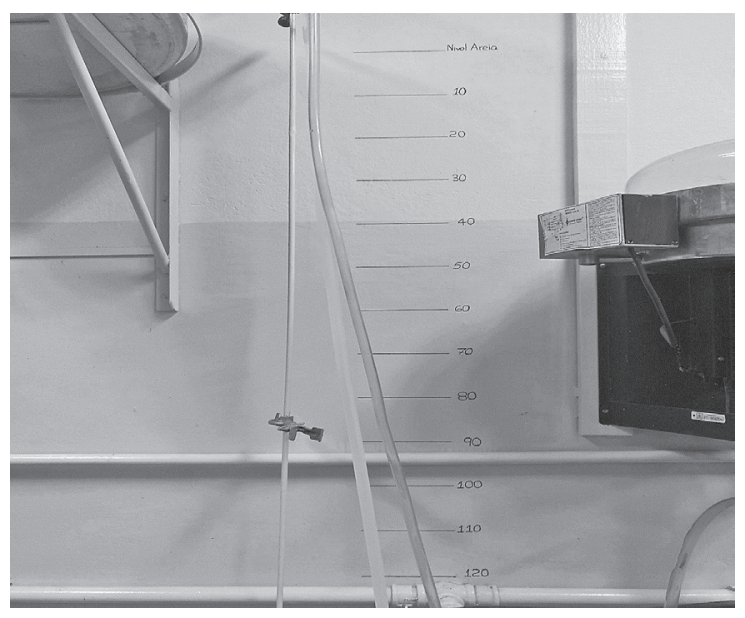

Figura 3. Diferentes niveles de tensión a aplicar expresado en centímetros de altura de columna de agua

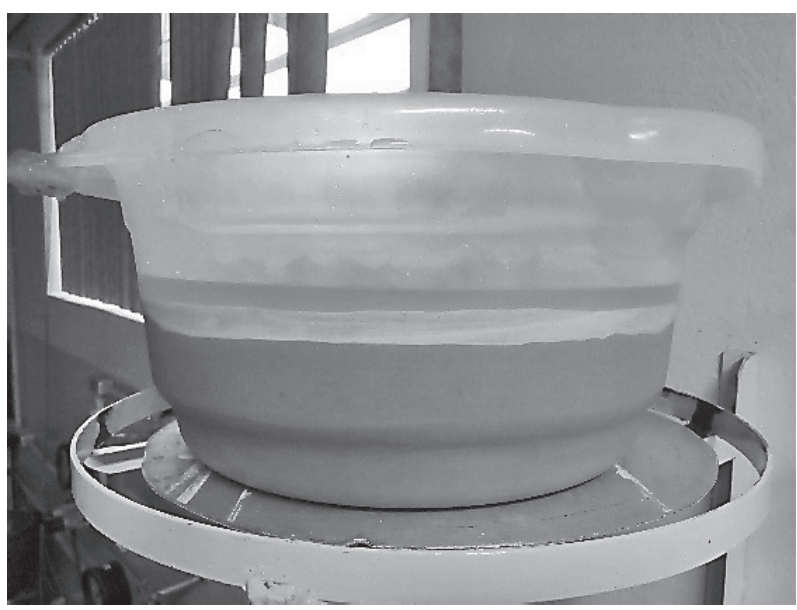

Figura 4. Colocación de los dos tamaños de arena para formar una doble capa de succión.

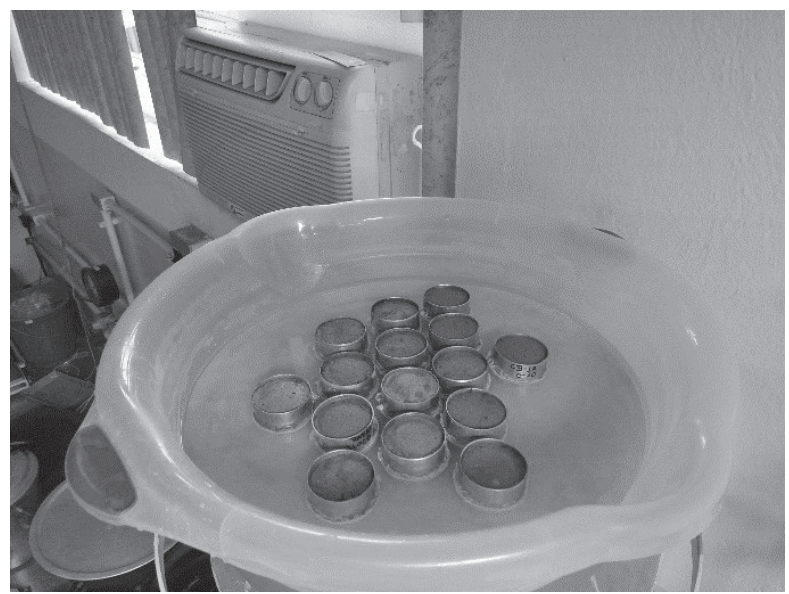

Figura 5. Colocación de las muestras en la mesa de succión para saturación y posterior proceso de aplicación de diferentes succiones 


\section{Resultado y sus análisis}

El promedio de las 3 repeticiones para cada una de las succiones con respecto a la humedad del suelo expresado en los porcentajes de humedad gravimétrica y humedad volumétrica se muestra en el cuadro 1

Cuadro 1. Porcentaje de humedad gravimétrica y volumétrica promedio presente en las muestras de suelo arenoso según la succión ejercida por la columna de agua.

\begin{tabular}{|c|c|c|}
\hline Tensión & $\begin{array}{c}\text { \%HG } \\
\text { Promedio }\end{array}$ & $\begin{array}{c}\% \text { HV } \\
\text { Promedio }\end{array}$ \\
\hline 0,0001 & 22,52 & 36,26 \\
\hline 10 & 21,45 & 34,53 \\
\hline 20 & 20,57 & 33,12 \\
\hline 30 & 19,01 & 30,61 \\
\hline 40 & 15,90 & 25,60 \\
\hline 50 & 12,05 & 19,40 \\
\hline 60 & 6,91 & 11,12 \\
\hline 70 & 5,52 & 8,88 \\
\hline 80 & 4,48 & 7,21 \\
\hline 90 & 3,92 & 6,32 \\
\hline 100 & 3,56 & 5,73 \\
\hline 110 & 2,45 & 3,95 \\
\hline 120 & 1,86 & 2,99 \\
\hline Dap & 1,61 & $9 / \mathrm{cm}^{3}$ \\
\hline
\end{tabular}

Se observa en el cuadro 1 que la microporosidad o la máxima capacidad de retención total de humedad en el suelo en contra de la fuerza de la gravedad que se define como la cantidad de agua que queda en el suelo cuando este ha sido sometido a una succión de $60 \mathrm{cmca}$ expresadas en términos de porcentaje de humedad gravimétrica $\left(\left(g_{\text {agua }} / g_{\text {suelo }}\right){ }^{*} 100\right)$ y porcentaje de humedad volumétrica $\left(\mathrm{cm}^{3}{ }_{\text {agua }} / \mathrm{cm}^{3}{ }_{\text {suelo }}\right)^{\star} 100$ son de $6,91 \%$ y $11,12 \%$ respectivamente. La microporosidad cuando el suelo ha sido sometido a una succión de 100 cmca expresadas en términos de porcentaje de humedad gravimétrica y porcentaje de humedad volumétrica son de $3,56 \%$ y $5,73 \%$ respectivamente. La macroporosidad o porosidad drenable expresada en términos de porcentaje de humedad gravimétrica y volumétrica cuando el suelo ha sido sometido a una succión de 60 cmca es de $15,61 \%$ y $25,14 \%$ respectivamente y la macroporosidad cuando el suelo ha sido sometido a una succión de $100 \mathrm{cmca}$ expresadas en términos de porcentaje de humedad gravimétrica y volumétrica es de $18,96 \%$ y $30,53 \%$ respectivamente. 


\section{Recomendaciones}

Para obtener mayor capacidad de succión de 120 cmca en la mesa de succión se debe de usar arena muy fina en la capa superior en el caso de tener escases de la misma, sino en su defecto; utilizar en toda la mesa de succión arena muy fina.

Las burbujas de aire deben ser en la medida de lo posible, eliminadas por completo del sistema de tuberías debido a que éstas rompen la columna de succión de agua que se ejerce sobre las muestras.

\section{Conclusiones}

La mesa de succión o mesa de tensión de bajo costo es una buena herramienta para determinar la macroporosidad del suelo llamada también porosidad drenable total del suelo según los valores teóricamente definidos. Además, es una herramienta para determinar microporosidad del suelo o capacidad de campo expresado en succiones de $60 \mathrm{cmca}$ o de $100 \mathrm{cmca}$.

No obstante, en suelos que presentan mucha macroporosidad como es el caso de los suelos arenosos y areno francosos, la mesa de succión funciona para determinar la curva característica de desabsorción de agua en el suelo donde se puede obtener porcentajes de humedades gravimétricas y volumétricas bajos en el suelo como el caso de 1,86\% de humedad gravimétrica correspondiente a 2,99\% de humedad volumétrica encontradas las cuales corresponden a porcentajes de humedad del punto de marchitez permanente según la literatura.

Ante lo anterior, este equipo presenta un costo bajo respecto al ofrecimiento de casas comerciales.

\section{Referencias}

[1] J. Porta, M. A. López and R. Poch, Edafología: Uso y protección de suelos, Cataluña: Mundi-Prensa, 2014, p. 608.

[2] M. Rijo, 2017.

[3] M. Calvache, Universidad Técnica de Manabí, Facultad de Ciencias Agropecuarias., 2002.

[4] A. L. Jordan, Manual de Edafología, Sevilla, 2016, p. 143.

[5] N. C. Brady and R. R. Weil, Elementos da naturaleza e propiedades dos solos, 3ra. ed., Porto Alegre: Bookman, 2013, p. 686.

[6] Empresa Brasileira de Pesquisa Agropecuária (EMBRAPA), Manual de métodos de análise de solo, 3ra. rev. e ampl. ed., Brasilia, D.F. Brasilia, 2017, p. 573.

[7] S. Nascimento Duarte, J. Honorio de Miranda, É. Farías de França e Silva, J. Francismar de Medeiros, R. Tábora Costa and H. Raj Greyi, Fundamentos de drenajem agrícola, 1ra ed., Fortaleza, 2015, p. 356.

[8] C. Rogério de Mello, G. César de Oliveira, D. Furtado Ferreira and J. María de Lima, "Predição da porosidade drenável e disponibilidade de água para Cambissolos da Microrregião Campos das Vertentes, MG.," Pesquisa Agropecuaria, vol. 37, no. 9, pp. 1319 - 1324, 92002.

[9] P. Silva C., H. Silva R., M. Garrido S. and E. Acevedo H., Santiago, 2015, p. 86.

[10] Unidad de hidrología, Dpto de Suelos y aguas, Facultad de Agronomía - Universidad de la repúbica, Montevideo, Sf., p. 45.

[11] Traxco, Componentes para sistemas de riego Pivot, 2009. 